\title{
A comprehensive up-to-date reference on ferrets
}

\author{
Reviewed by James R. Fahey, MS, PhD, DVM, DACVM
}

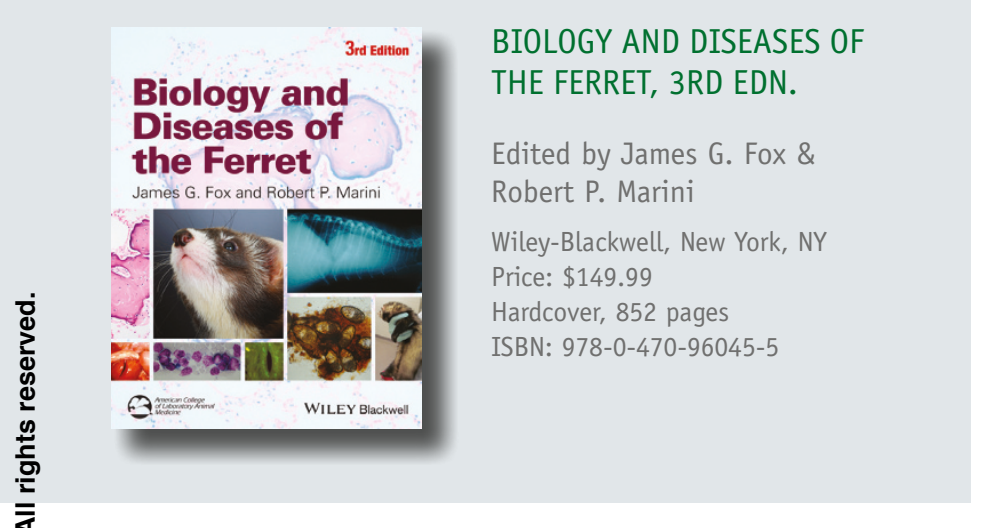

The third edition of Biology and Diseases of the Ferret is the latest version of this classical text about members of the genus Mustela, small carnivores that have great importance as research models, as household pets and, in the case of the black-footed ferret, as endangered species. The book, which was last revised in 1998, has been thoroughly updated in 2014. It includes ten new chapters and has been restructured to devote full chapters to the subjects of surgery, anesthesia and biomethods, which were previously combined into one chapter. This book is encyclopedic in its coverage of ferret biology, medicine and research. Here I have highlighted selected chapters from each section of the book as examples of its valuable content.

The newly revised book is organized into three sections: Biology and Husbandry, Diseases and Clinical Applications, and Research and Applications. The first section (chapters 1-10) covers the essentials of ferret biology. The first chapter, "Taxonomy, History and Use," gives an instructive summary of the evolution of the relationship between humans and ferrets over time, from early use of ferrets as rodent eradicators to current-day use of ferrets in biomedical research. For the modern-day facility veterinarian, chapter 6 provides important considerations for the housing and husbandry of ferrets in animal facilities. Additionally, there is a section on housing specific pathogen-free ferrets for use in infectious disease research and, for those who keep ferrets as pets, a description of the basic necessities for keeping ferrets at home. Chapter 9 provides a concise reference for the US and European regulatory guidelines for space allowance, care and use of ferrets as research animals and the regulations regarding ferrets as companion animals. This is particularly important information for individuals and institutions considering the acquisition of ferrets. Chapter 9 also outlines ferret transportation information, including online links about animal

Fahey is Chief of Diagnostic Services/Associate Direction, Laboratory Animal Health Services at The Jackson Laboratory in Bar Harbor, ME. transport and a table of the required documents for transporting laboratory animals. Chapter 10 provides natural history information on the black-footed ferret, an endangered species native to North America. Of particular interest is the description of reproductive science and genetic studies that have taken place as part of the effort to save and repopulate this important species of ferrets.

The second section of the book, Diseases and Clinical Applications (chapters 11-24), reports up-to-date information about biomethodology, surgery and anesthesia, body systems diseases and infectious diseases of ferrets. The first three chapters of this section (chapters 11-13) contain a wealth of information for clinical veterinarians and scientists who use ferrets in research. In chapter 11, there is broad coverage of biomethods, including physical exam, restraint, blood collection, imaging methods, urinary catheterization and vaccination of ferrets as research animals. The chapter also includes a useful table of vaccinations and preventive medicine elective procedures. Similarly, chapter 12 gives valuable descriptions of methods for administration of analgesics and anesthetics as well as information on specific drug selections. I found the tables with drug types, dosages and duration of effects to be particularly useful. Chapter 13 provides details on hemostasis, useful surgical materials and suitable details on specific body system surgical procedures. The coverage of body system diseases in chapters $14-19$ is wideranging and reviews clinical signs, diagnostics, treatments and other considerations of disease conditions in the hematopoietic, genitourinary, gastrointestinal, endocrine and cardiovascular systems. Additionally, chapter 19 summarizes information on diseases of the central nervous, eye, musculoskeletal and respiratory systems. There is also a brief overview of systemic toxicoses. In total, this section is a commendable assemblage of medical knowledge that veterinarians and research scientists will appreciate.

The third section of the book (chapters 25-32) is devoted to current research trends with ferret models of disease. The section reviews viral respiratory diseases and Morbillivirus infections, cardiovascular research, genetic engineering, hearing and auditory function, visual system development and plasticity, nausea and vomiting research and its translation to clinical application, lung carcinogenesis and nutritional chemoprevention research. The information is largely new with many literature citations from recent years. Additionally, each chapter features excellent photographs, graphic illustrations and tables relevant to the subject at hand.

I highly recommend this book for everyone who works with ferrets in a research setting because it is comprehensive, up-to-date and highly informative. Furthermore, I would also recommend the book as an excellent reference text for veterinarians in non-research clinical practice and for those who have a general interest in the biology and medicine of ferrets. 